\title{
On the Potential of the Excluded Volume and Auto-Correlation as Neuromorphometric Descriptors
}

\author{
L. da F. Costa, M. S. Barbosa ${ }^{a, *}$ and V. Coupez ${ }^{\text {b }}$ \\ ${ }^{a}$ Cybernetic Vision Research Group Instituto de Física de São Carlos University of \\ São Paulo 13560-970 São Carlos, SP, Brazil \\ b Polytechnic Institute of Orléans, ESPEO. 12, rue de Blois. BP 674445067 \\ Orléans, Cedex 2, France
}

\begin{abstract}
This work investigates at what degree two neuromorphometric measurements, namely the autocorrelation and the excluded volume of a neuronal cell can influence the characterization and classification of such a type of cells. While the autocorrelation function presents good potential for quantifying the dendrite-dendrite connectivity of cells in mosaic tilings, the excluded volume, i.e. the amount of the surround space which is geometrically not accessible to an axon or dendrite, provides a complementary characterization of the cell connectivity. The potential of such approaches is illustrated with respect to real neuronal cells.
\end{abstract}

Key words: Morphometry, Neural Networks.

PACS: 87.80.Pa, 87.19.La

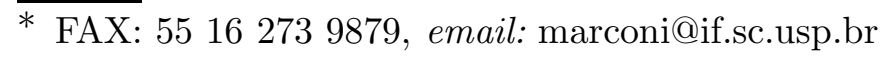

Preprint submitted to Elsevier Science

14 November 2018 


\section{Introduction}

Neuroanatomy is, in many aspects, a key factor influencing the overall nervous system performance, regarding health and adaptation, underlined by the spatial characteristics of neural networks and by the geometry of their building blocks, the neuronal cells. The function of such assemblage of cells, which integrates synaptic propagation of action potentials, has long been related to the shape of the individual cell and specific dendritic morphology [1]. The study of morphological characteristics of neuronal cells, although overshadowed by the flood of advances in neurophysiological explorations along the last decades, was resumed in the seventies as an approach in which computer science methods provide the means for quantifying, in an objective way, morphological subtleties that would otherwise go unnoticed by the human eye [2]. Examples of geometrical features commonly used in computational neuromorphology include the influence area, number of dendritic segments and bending energy (see [3] for an extensive list) and as well as standard and multi-scale fractal analysis $[4,5,6,7,8,9]$. It has also been shown more recently that neuronal cell characterization can be efficiently performed with basis on multi-scale shape functionals derived from integral geometry and applied to a database containing two classes of cat ganglion cells [10].

In the current work we extend geometrical features that are not only useful for neuromorphic characterization, but which are also more closely related to the functionality of the network, with emphasis on their abilities at making connections as highlighted in $[11,12,13]$. In particular, we explore and assess the use of the autocorrelation function and excluded volume of the shape of a neuronal cell as meaningful measurements to be used in cell characteriza- 
tion, classification and simulation. The reader is reported to $[14,15,16]$ for pioneering works related to these concepts. It is shown here that the autocorrelation function quantifies in a precise fashion the potential of the cell for dendrite-dendrite connections, a situation that is especially relevant for neuronal systems involving electrical synapses. At the same time, the excluded volume is verified to provide additional information about the cell potential for connectivity, but now from the complementary perspective of unreachable portions of the arborizations.

The article starts by presenting in Section 2 the suggested measurements and by describing their respective application to a few real ganglionary cells. Section 3 presents our results for an extended database of neuronal cells containing 50 cells, showing the discriminative potential of the proposed methodology. Section 4 is dedicated to concluding comments.

\section{Shape Descriptors}

This section describes the two considered neuronal features, namely the twodimensional autocorrelation function and the excluded volume. These measures have in common the conceptual advantage of being related to the connectivity potential of the cell in an actual simulation of a morphological neuronal network. Although the excluded volume and the autocorrelation function are not multi-scale functionals, the former situation demands more intense computational efforts when compared to the latter which is faster as its implementation can be done by using properties of Fourier analysis. 


\subsection{Autocorrelation Function}

Given a function $\varphi(x, y)$, its autocorrelation $\alpha(x, y)$ is defined as in Equation 1, which can be effectively evaluated by using the Fourier transform (Equation 2).

$$
\begin{array}{r}
\alpha(x, y)=\int_{-\infty}^{\infty} \int_{-\infty}^{\infty} \varphi(r, s) \varphi(r+x, s+y) d r d s \\
\alpha(x, y)=\mathcal{F}^{-1}\left(\mathcal{F}(\varphi(x, y)) \mathcal{F}\left(\varphi(x, y)^{*}\right)\right.
\end{array}
$$

Let the spatially sampled neuronal cell morphology (dendrites and soma) be represented in terms of a binary image, with each cell point represented as 1 and taking the center of mass of the respective soma (i.e. cell body) as the coordinate system origin. The autocorrelation of this shape at a specific point $(x, y)$, i.e. $\alpha(x, y)$ corresponds to the number of intersection points between two versions of the cell with relative displacement of $(x, y)$. Therefore, the autocorrelation function quantifies the cell potential for dendrite-dendrite connections with respect to copies of itself placed at all possible relative positions, see Figure 1 for examples of the autocorrelation for few prototypical shapes. Figure 2 illustrates the autocorrelation function for a series of cat ganglion cells. It is important to observe that the information provided by the autocorrelation analysis of the cell morphology represents a second order analysis of the cell geometry, which could not be otherwise obtained by more traditional measurements directly focusing the cell, such as its area, perimeter, and so on.

The autocorrelation function is immediately related to the two following biological questions: (a) in a relatively homogeneous morphometric network (i.e. composed by similar copies of the same cell) is there a preferential orienta- 
tion where the cell presents highest or lowest potential for connection? (b) the same question regarding radial distance. Given a neuronal cell and its respective two-dimensional autocorrelation function, it is therefore interesting to derive some particularly meaningful one-dimensional signatures such as the functions defined in Equations 3 and 4.

$$
\begin{aligned}
& f(\theta)=\int_{r \in L(\theta)} \alpha(x, y) d r \\
& g(r)=\int_{\theta \in C(r)} \alpha(x, y) d \theta
\end{aligned}
$$

where $L(\theta)$ is the line segment starting at the coordinate system origin and making an angle of $\theta$ with the $x$-axis, $\theta \in[0,2 \pi)$, and $C(r)$ is the circle of radius $r$ centered at the coordinate system origin. While the function $f(\theta)$ can be understood as the potential of the cell to make dendrite-dendrite connections in terms of the angle $\theta$ made with the $x$-axis, the function $g(r)$ gives the potential for connections in terms of the distance $r$ from the soma center of mass.

\subsection{Excluded Volume}

The excluded volume for a given object is usually defined as the volume surrounding and including a given object, which is excluded to another object as it is placed at varying relative positions $[17,18]$. A similar definition of the excluded area holds in 2-D. Although we henceforth refer to this concept as excluded "volume", it is important to keep in mind that it actually refers to an area measurement taking into account the topological features of the cell. At the same time, 3D generalizations are also possible and interesting and will be considered in future works. This functional is always defined for a pair of 

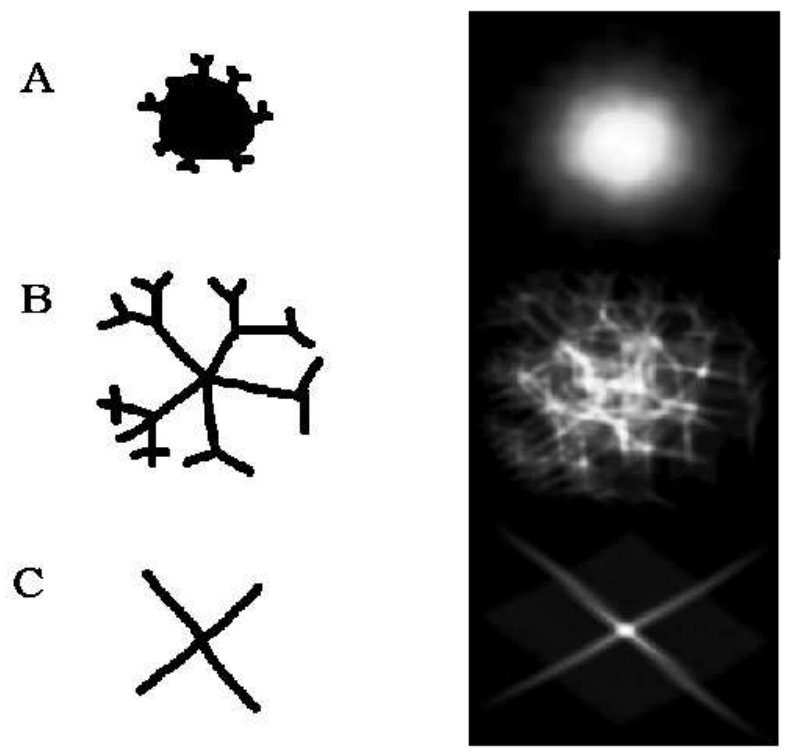

Fig. 1. The autocorrelation function for prototypical different cells.

objects.

The excluded volume terminology comes from the statistical mechanics of nonideal gases, where this functional arises in the leading order concentration expansion (virial expansion) for the pressure in the case of gas particles that repel each other with a hard-core volume exclusion [19]. This idea is frequently employed also in systems that share the same physical constraints such as colloidal solutions in polymer sciences.

The general expression for the excluded volume $V_{e x}$ of two convex objects, involving their surface area $F_{i}$ and mean radius of curvature of each $R_{i}$ and their individual volumes, $V_{i}$, is given by (see [19]):

$$
<V_{e x}>=V_{1}+V_{2}+\left(A_{1} R_{1}+A_{2} R_{2}\right) /(4 \pi)
$$



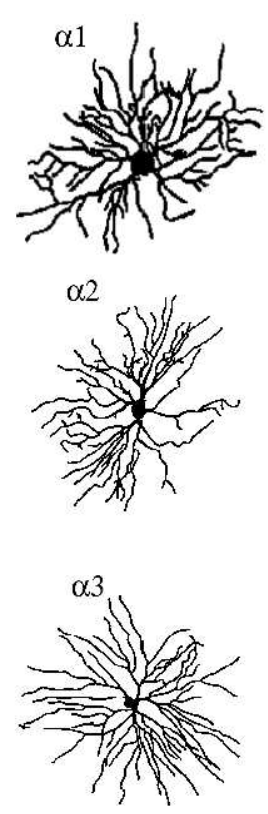

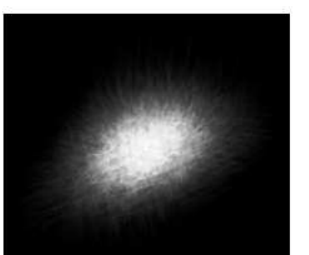

$\beta 1$
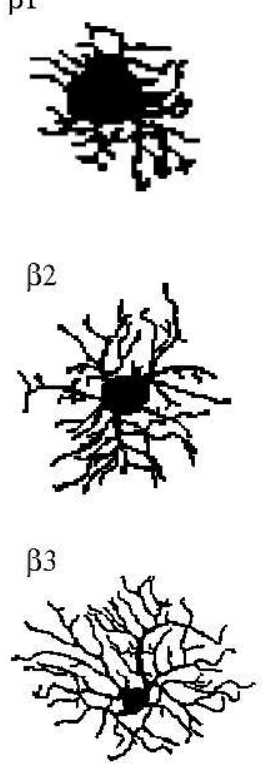

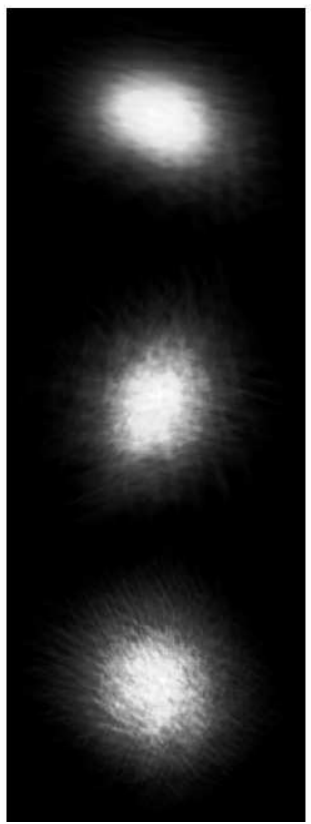

Fig. 2. The autocorrelation function of typical ganglion cells of the domestic cat retina from two phyisiological class. The alpha and beta types are shown at the left and right-hand sides, respectively. The neuronal cell images were adapted with permission from [2].

Here we evaluate a computational variant of the excluded volume concept. More specifically, our algorithm tries to fit a copy of the cell shape at every relative position on an orthogonal grid, while checking for intersections. Whenever no intersection is found, the respective region below the image is considered accessible, and the excluded volume function at that specific point is marked as 0 , otherwise it is marked as 1 . Figure 3 illustrates the excluded volume function obtained for several different cells.

It stems from the above definition that all convex shapes have a null excluded volume. For an intricate geometry such as neuronal cell forms this functional can be understood as an indication of the boundaries of connectable portions of the cell. From the functional point of view, all dendrites which are trapped 
inside an excluded volume region will not actually establish connections with other cells, compromising the performance of a network made with copies of that same cell. This fact is being further investigated as a part of an ongoing research on morphological Hopfield networks [20].
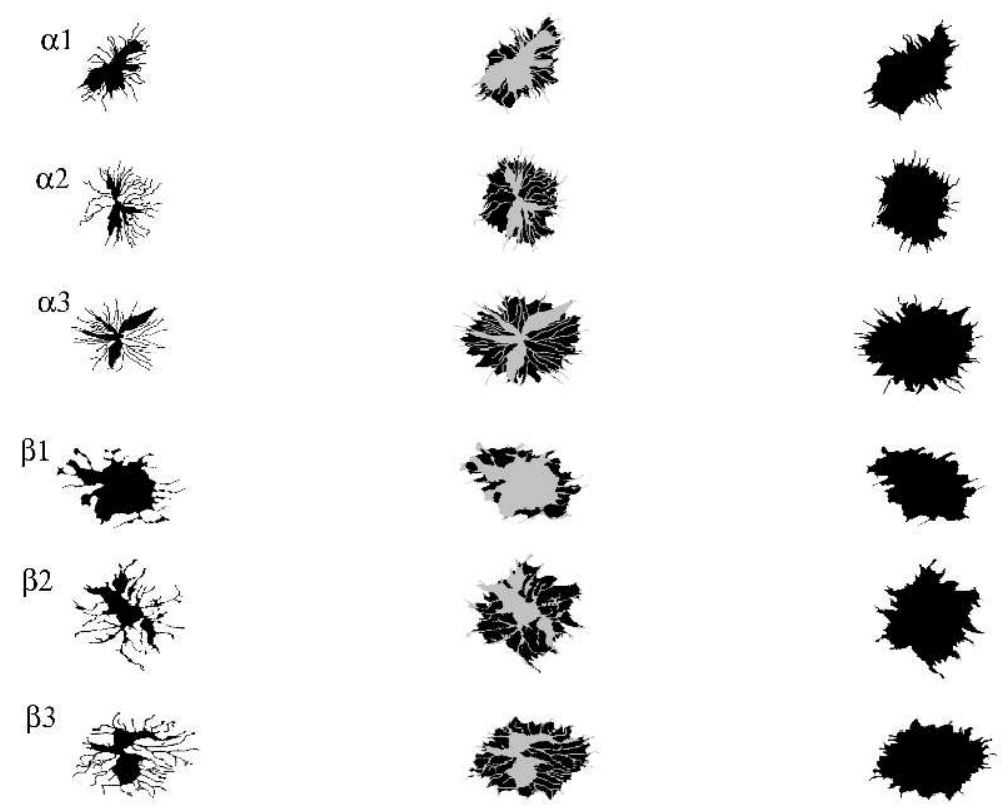

Fig. 3. The excluded volume for examples of real ganglion cells of the domestic cat retina. The neuronal cell images were adapted with permission from [2].

\section{Results and Discussion}

In this work we use a database of 53 images of ganglion cells from the domestic cat retina of which 26 are of type alpha and the remaing 27 of type beta. These two types of neuronal cells can often be difficult to be distiguished by human visual inspection. Typical cells from these classes are illustrated in Figures 2 and 3 . 
Figures 4 and 5 present the functions $f(\theta)$ and $g(r)$ (Equations 3, 4) for the ganglion cells in Figure 2. These especially representative 6 cells were chosen for illustrative purposes, as the functions $f(\theta)$ and $g(r)$ would result too cluttered in case all 53 cells were used. It is clear from the first of these figures that quite different curves are obtained for each neuronal class, suggesting that the considered measurements have good discriminative power. On the other hand, the curves obtained for the autocorrelation line integrals in terms of the radial distance, shown in Figure 5, are characterized by similar overall behaviour, but different magnitudes. Although such a result implies less discriminative power for this measurement, it also represents an interesting biological phenomenon on its own, indicating that the considered cells share quite similar potential for connections as far as the radial distance is concerned. More specifically, such curves are characterized by small values at both left and right-hand extremities, taking a maximum value in its intermediate portion. While the small values for small distances is expected since line integrals for those cases cover less perimeter, the small values obtained for large radial distances is a consequence of the progressive termination of the dendritic extremities. It remains to be further investigated whether similar curves are obtained for classes of neuronal cells other than ganglion retinal neurons.

Figure 6 shows the phase space or scatter plot, defined by the standard deviation of the correlation integral for angles and the excluded volume, obtained for the considered ganglion cells. It is clear from this figure that the two classes of cells are well-separated conforming to the discriminative potential of the former of these features. At the same time, this same figure indicated that, at least for the considered data, the excluded volume has less discriminative power, despite of its direct relationship with the connectivity potential 
of a cell. This could be related to the fact that the measure is not multiscale. Work to extend this notion and to extract more information of this important concept is in course.

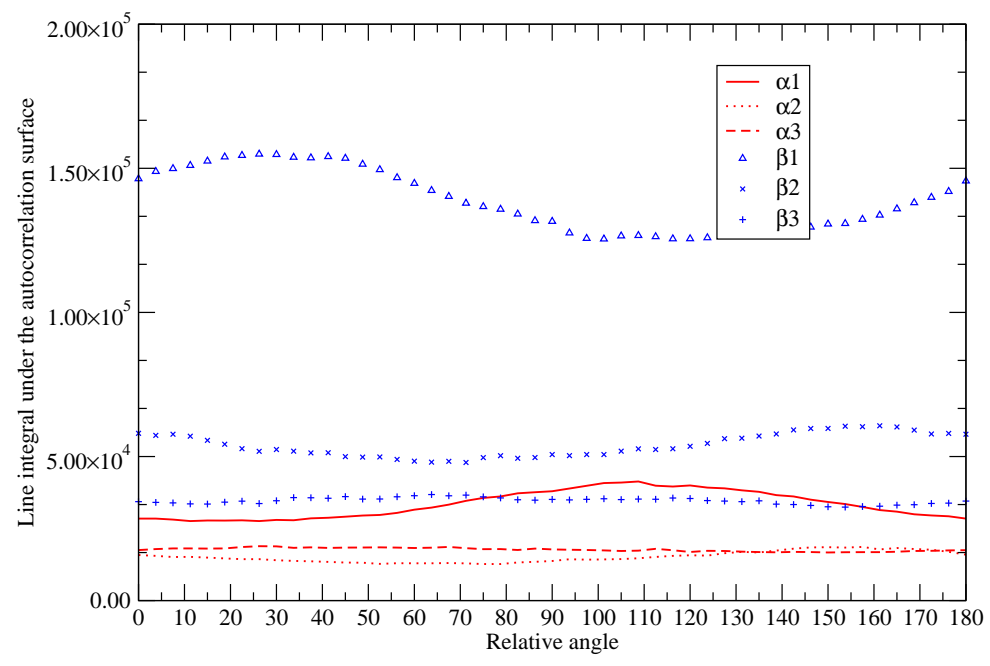

Fig. 4. The line integral of the correlation function in terms of the angle of a line passing through the maximum point of correlation. In this figure we show results for typical cells of the two physiological classes of cat ganglion cells shown in figure 2 .

Table 1

\begin{tabular}{|c|c|c|c|c|c|}
\hline & $\alpha$ & $\beta$ & Error & Posterior.Error & Stratified.Error \\
\hline$\alpha$ & 25 & 1 & 0.0384615 & -0.1151105 & -0.0437544 \\
\hline$\beta$ & 5 & 22 & 0.2380952 & 0.2949808 & 0.2197885 \\
\hline Overall & & & 0.1382784 & 0.0899351 & 0.0880171 \\
\hline
\end{tabular}

The result of classical discriminant analysis for the measures considered in the scatterplot of Figure 6. From this cross-validation table we observe a good discriminative potential, most of it derived (see Figure 6) from the autocorrelation functionals. 


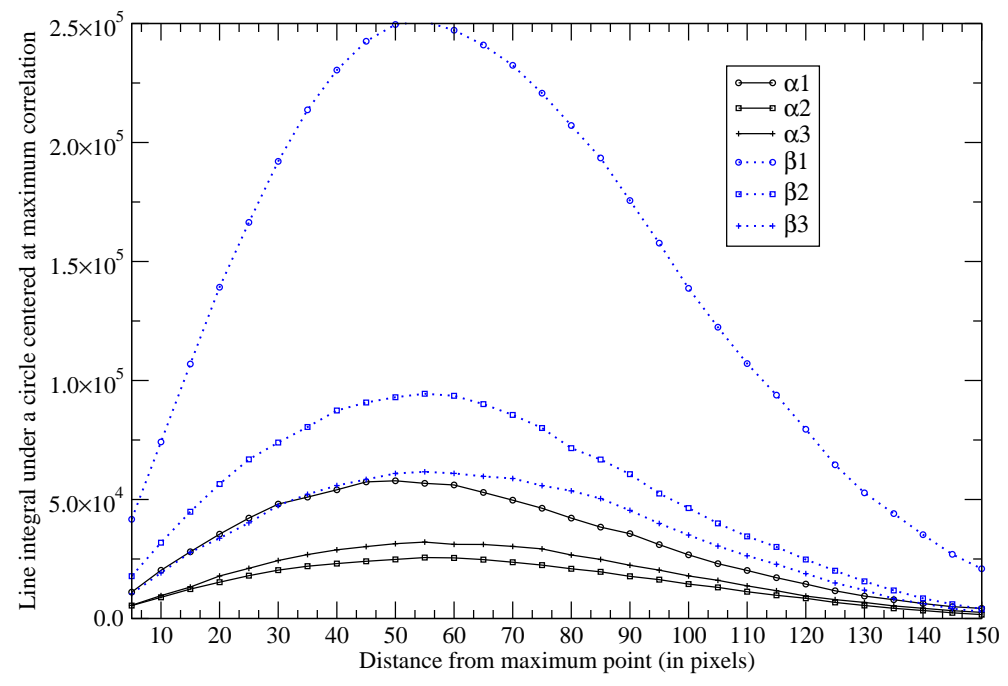

Fig. 5. The line integral of the correlation function in terms of the radius of a circle centered at the maximum point of correlation. In this figure we show results for the same typical cells of the domestic cat ganglion cells shown in figure 2 .

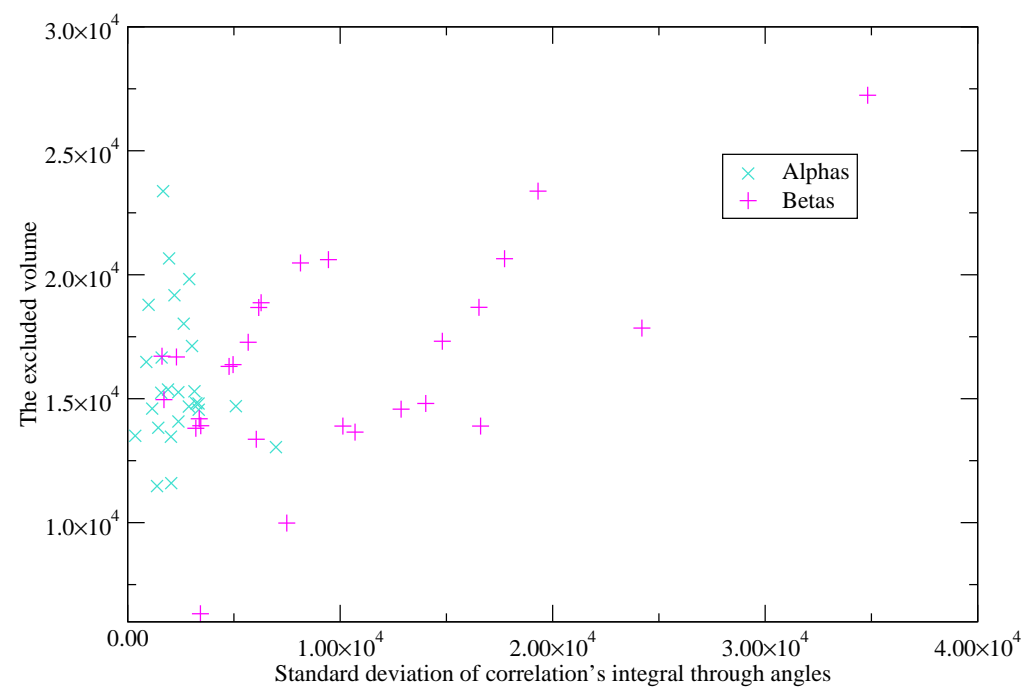

Fig. 6. A scatter plot, defined by measures of excluded volume and auto correlation, exhibiting significant clustering of two physiological classes of cat ganglion cells. 
Table 1 shows the result of statistical discriminant analysis [21] for all the 53 considered cells. This cross-validation table shows clearly the potential of the proposed methodology for proper cell classification. While the classification of $\alpha$ cells was particularly effective with just one cell out of 26 misclassified, the identification of the beta cells was relatively poorer with 5 out of the 27 cells being misclassified as alpha type. This is in part a consequence of the fact that the cells are difficult to be separated even by visual inspection.

Despite a few misclassified cells, the suggested measurements were verified to be capable of discriminating between the 53 considered cells. However, it is important to bear in mind that the importance of the suggested neuromorphometrical features goes beyond such an ability for cell discrimination, in the sense that it reflects in a quantitative way the potential of the investigated cells to establish synaptic contacts, which is an all important property in biological neuronal systems. In addition, the excluded volume provides additional information about the spatial delimitation of the neuronal processes, which is also important in defining cell connections. In this way, the suggested methodology allows the prediction of macroscopic properties of the neuronal system with basis on local geometrical features of individual representative cells.

\section{Concluding Remarks}

This work presented a preliminary assessment of the potential for neuromorphometric characterization of two features directly related to the ability of a neuronal cell to make dendrite-dendrite connections, which is immediately

relevant for defining and constraining the behaviour of neuronal systems. In particular, we have shown that the autocorrelation function leads to different 
profiles for the cells in the considered ganglion cell database. The extracted features resulted particularly efficient for cell classification. When taken on its own, the excluded volume led to poorer discriminative potential. The continuation of this work should include the correlation of the suggested measurements with functionals expressing the performance of morphological neuronal networks [20] as well as other shape functionals [10,13], 3D and multiscale extensions of those measurements. Another interesting perspective that is being investigated is how the proposed measurements and methodology, which implies neuronal networks composed by identical copies of the same cell, can be extended according to a mean-field approach, where a mean representation of the cell morphology is used to analyse networks composed by cells with varying morphology.

\section{References}

[1] S. R. Cajal. Textura del sistema nervioso del hombre e y los vertebrados. Oxford University Press, 1990. Translated by N. Swanson and L. W. Swanson.

[2] B. B. Boycott and H. Wassle. The morphological types of ganglion cells of the domestic cat's retina. J. Physiol, 240:397-419, 1974.

[3] L da F. Costa and T. J. Velte. Automatic characterization and classification of ganglion cells from the salamader retina. Journal of Comparative Neurology, 404(1):33-51, 1999.

[4] K. Morigiwa, M. Tauchi, and Y. Fukuda. Fractal analysis of ganglion cell dendritic branching patterns of rat and cat retinae. Neurosci. Res., 10:131-9, 1989. 
[5] E. Fernandez and H. F. Jelinek. The use of fractal theory in neuroscience: Methods, advantages and potential problems. Methods, 24(4):309-321, 2001.

[6] C. L. Jones and H. F. Jelinek. Wavelet packet fractal analysis of neuronal morphology. Methods, 24(4):347-358, 2001.

[7] R. C. Coelho and L. da F. Costa. On the application of the bouligand-minkowski fractal dimension for shape characterization. Applied Signal Processing, 3:163$176,1996$.

[8] L. da F. Costa, E. T. M. Manoel, F. Fauchereau, J. Chelly, J. van Pelt, and G. Ramakers. A shape analysis framework for neuromorphometry. Network: Comput Neural Syst, 13:283-310, 2002.

[9] J. T. J. Smith, G. D. Lange, and W. B. Marks. Fractal methods and results in celular morphology-dimensions, lacunarity and multifractals. Journal of Neuroscience Methods, 69:123-136, 1996.

[10] M. S. Barbosa, E. S. Bernardes, and L. da F. Costa. Neuromorphometric characterization with shape functionals. Phys. Rev. E, 67:061910, 2003.

[11] O. Shefi et al. Morphological characterization of in vitro neuronal networks. Phys. Rev. E, 66(021905), 2002.

[12] S. Morita et al. Geometrical structure of the neuronal network of caenorhabditis elegans. Physica A, 298:553, 2001.

[13] L. da F. Costa and E. T. M. Manoel. A percolation approach to neural morphometry and connectivity. Neuroinformatics, 1:66, 2002.

[14] R. W. Rodieck. The density recovery profile: a method for the analysis of points in the plane applicable to retinal studies. Visual Neurosciences, 6:95-111, 1981.

[15] J. E. Cook. Spatial properties of retinal mosaics: an empirical evaluation of same existing measures. Visual Neuroscience, 13:15-30, 1996. 
[16] H. Wässle, L. Peichl, and B. B. Boycott. Dendritic territories of cat retinal ganglion cells. Nature, 292:344-345, 1981.

[17] L. A. Santalo. Integral geometry and geometrical probabilities, volume 1 of Encyclopedia of Mathematics. Addison Wesley, New York, 1976.

[18] L. Onsager. The effects of shape on the interation of colloidal particles. Ann. N.Y. Acad. Sci., 51:627, 1949.

[19] A. Isihara. Determination of molecular shape by osmotic measurement. $J$. Chem. Phys., 18:1446, 1950.

[20] L. da F. Costa, M. S. Barbosa, V. Coupez, and D. Stauffer. Morphological Hopfield networks. Brain and Mind, 4:91-105, 2003.

[21] G. J. McLachlan. Discriminant Analysis and Statistical Pattern Recognition. John Wiley \& Sons, 1992. 